\title{
A novel approach in cardiac surgery: Neifer's approach
}

\author{
Chaouki Neifer* \\ Rangueil University Hospital, Toulouse, France; Faculty of medicine, Sfax University, Tunisia
}

\begin{abstract}
While cardiac surgery has well evolved in the recent decades, endoscopic access to the heart is yet involving pleura opening and transcatheter procedures are still implying X-rays use. Here I introduce a novel minimally-invasive approach based on a sternal trepanation with extrapleural intercostal port(s). This allows a totally endoscopic access to the cardiac cavity without pleura opening. Besides, using this approach with live computer simulation, transcatheter procedures will be exclusively monitored by transesophageal echocardiography without X-rays need. Thus, current indications and practices in both cardiac surgery and interventional cardiology may be affected. Until effectiveness confirmation by experiments which are still in progress, a theoretical description of this innovative technique and its potential applications is detailed in this paper.
\end{abstract}

Abbreviations: 3D-TEE: Three-Dimensional Transesophageal Echocardiography; TAVI: Transcatheter Aortic Valve Implantation; URPV: Upper Right Pulmonary Vein

\section{Introduction}

From the end of $19^{\text {th }}$ century until now, access to the heart is allowed by either sternotomy or thoracotomy, with variations of incisions site and length [1]. Technology evolution has permitted to introduce endoscopic and robotically-assisted techniques in the cardiac surgery [2]. However, the used approach -intrapleural intercostal incisions- has not changed compared to the first report of Hans Christian Jacobaeus in 1909 [3]. Here I introduce a novel endoscopic approach for cardiac surgery using sternal trepanation with extrapleural intercostal small incision(s). While experiments are still in progress, I describe in this paper the theoretical approach with comments referring to recent literature.

\section{Surgical technique}

Basis of my approach is a direct access to the heart trough extrapleural intercostal port(s), key to this is to make first a sternal hole that will serve also as an additional working port and/or a lighting port using a special shape-adjusted camera. Several potential applications are allowed by this approach.

\section{Surgical pericardial drainage}

We make an infra-centimetric skin incision in the lower extremity of sternum body. Subcutaneous tissue is then incised with an electric scalpel down to the sternum. Then we proceed to 10 -mellimeters sternal trepanation using an auto-stop electric cranial drill. Pericardium is then easily opened with scissors or electric scalpel and pericardial liquid is aspirated. From the sternal hole, we introduce a curved tool to make a small extrapleural intercostal incision between the right edge of sternum and the internal thoracic vessels. This will be used to introduce optics if we want to take a look at the rest of cardiac cavity, to introduce another tool if we want to perform pericardial biopsies, and finally to introduce drainage tube (Figure 1).

\section{Epicardial pacemaker implantation}

We use the same approach as described above; sternal trepanation is however performed a bit higher. This allows us to implant pacemaker leads at the right ventricle and/or right atrium. Left chambers leads may be implanted by adding a left extrapleural intercostal small incision as described for the right one. Cables are then tunnelled to the pacemaker case through the intercostal incision(s).

\section{Central left ventricular unloading on extracorporeal life support}

The sternal hole in this case is made in the middle of sternum body. Then we add a right extrapleural intercostal port as described above and open pericardium. Through the sternal hole we spread the right atrium. The upper right pulmonary vein (URPV) is exposed and punctured with the discharge cannula introduced through the intercostal port. Cannula is then fixed to the skin. A second extrapleural intercostal working port is added if necessary. For decannulation, the URPV site puncture may be closed using the previous incisions or through sternotomy at moment of heart transplant or long-term cardiac support intervention.

\section{Transaortic Transcatheter Aortic Valve Implantation (TAVI)}

The sternal hole is made in the upper extremity of sternal body. One or two right extrapleural intercostal port(s) is (are) made as described above. We can add a suprasternal mini-incision to introduce optics, suction cannulas and the valve delivery system. After opening pericardium, we make two concentric pledgeted aortic purse-strings sutures, $2 \mathrm{~cm}$ proximal to the innominate artery. Depending on aorta conformation, aortic puncture and wires introduction will be allowed either through the sternal or the intercostal port(s). The valve delivery system may be introduced through the suprasternal incision. By this approach, the whole procedure may be performed without X-rays use: heart pacing is allowed by direct temporary epicardial right ventricle electrodes implantation, wires and valve introduction are exclusively

Correspondence to: Chaouki Neifer, Rangueil University Hospital, Toulouse, France; Faculty of medicine, Sfax University, Tunisia, Tel: 0033618222801, 0021652250510; E-mail: chawkineifar@yahoo.fr

Key words: cardiac surgery, endoscopic, minimally invasive, novel approach

Received: July 22, 2017; Accepted: August 28, 2017; Published: August 30, 2017 
monitored by three-dimensional transesophageal echocardiography (3D-TEE) aided by live computer simulation from preoperative data (CT measurements of ascending aorta, aortic valve, and coronary ostia; real measurements of wires and valve delivery system) (Figure 2).

\section{Heart valves repair}

I describe succinctly access to each valve; cardiopulmonary bypass and aorta endoclamping are allowed by peripheral cannulation.

Aortic valve (and pulmonary valve): Through a sternal hole made in the upper third of sternum body, we make four concentric extrapleural intercostal incisions as shown in Figure 3. Then we open and spread pericardium using tracting wires passed through the intercostal holes. Ascending aorta and pulmonary artery are then perfectly exposed. A suprasternal mini-incision may be useful for suturless valve introduction along aorta axis. By this approach we can treat aortic valve disease, ascending aorta aneurysm, and proximal pulmonary embolism.

Tricuspid valve (Figure 4): Access to tricuspid valve is allowed by one sternal hole in the lower third of sternum body and four concentric extrapleural intercostal holes. After opening pericardium, right atrium is incised and spread using tracting wires passed through the intercostal holes. Tricuspid valve is then perfectly exposed. By this approach, we can treat tricuspid valve disease and atrial septal defects.

Transcatheter tricuspid valve repair and transcatheter closure of atrial septal defect may be allowed by direct puncture of the right atrium, after preparing an atrial purse-strings suture that we close at the end. We use for that a sternal hole and only one or two right intercostal port(s) as described above. The whole procedure may be totally managed without X-rays use thanks to $3 \mathrm{D}$-TEE aided by live computer simulation.
Mitral valve: Using the same approach for the tricuspid valve, we add an interatrial septal incision that we spread alike by tracting wires. Mitral valve is then well exposed. By this approach, we can treat mitral valve disease and close left atrium.

Transcatheter mitral valve repair/replacement may also be managed by direct puncture of the right atrium and the interatrial septum. The resulting atrial septal defect is closed by a septal closure device that we introduce through the right atrium. The site puncture of the right atrium is closed using a purse-strings suture as described for the tricuspid valve approach. In fine, the whole procedure can be exclusively monitored by $3 \mathrm{D}-\mathrm{TEE}$ and live computer simulation, without $\mathrm{X}$-rays use.

\section{Comments}

Endoscopic approaches have invaded practically all surgical specialities offering the advantage of being less invasive with better postoperative recovery and aesthetic benefit. Despite some success in cardiac surgery [4], the evolution of these approaches remains poor in this speciality. Two reasons may explain this fact: first the complexity of the surgery itself demanding a long learning curve that cannot tolerate any mistake, secondly the need -in the video-assisted surgery- to open pleura and exclude lungs with potentially additional respiratory morbidity.

The technique I am introducing today allows an easy endoscopic access to the cardiac cavity without pleura or sternum opening, offering a good exposure for each indication with the minimum of incision morbidity.

Pericardial drainage is actually managed by either interventional cardiology demanding echo-guidance and/or fluoroscopy or cardiac

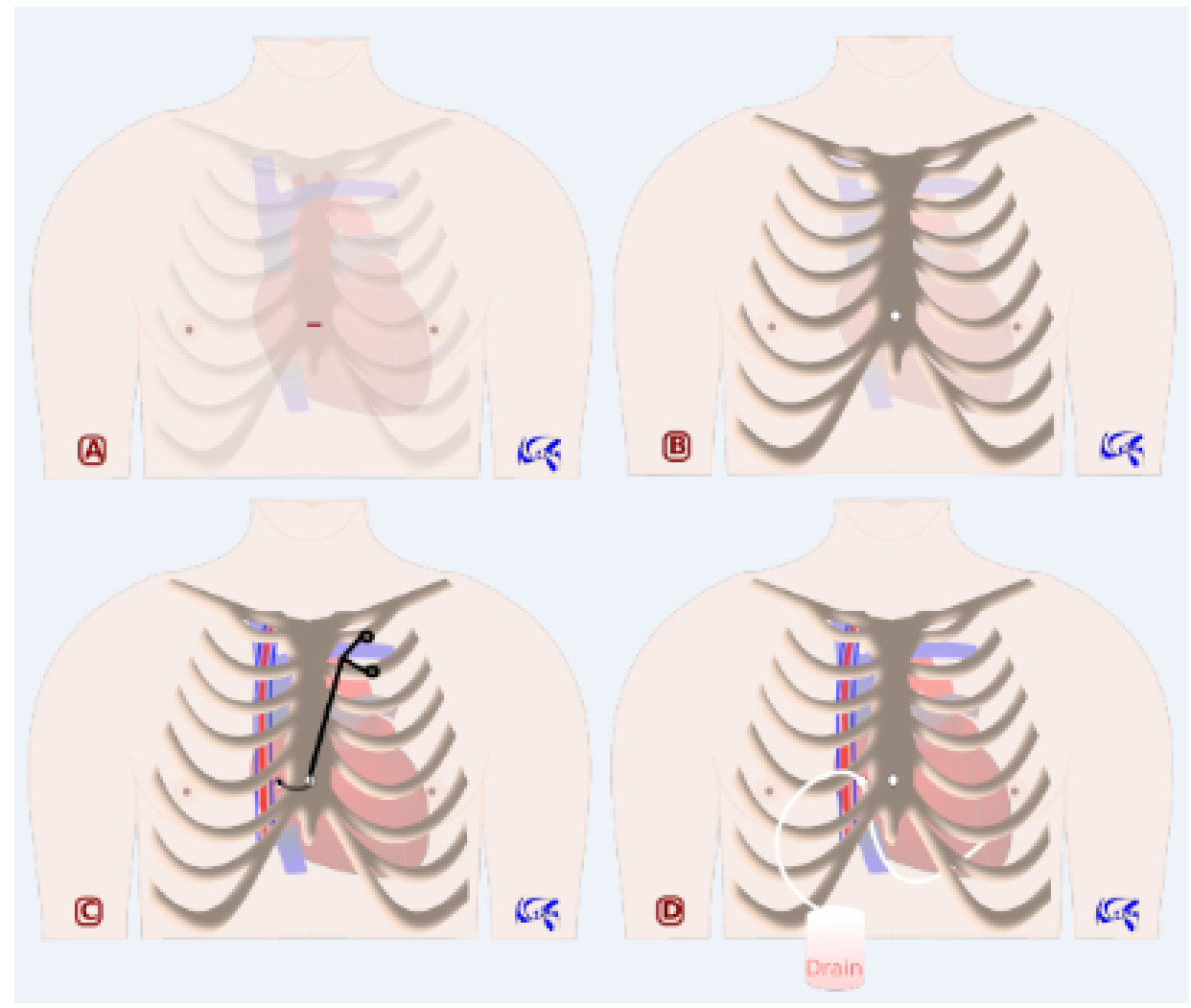

Figure 1. Neifer's approach for pericardial drainage. 1A: Incision site in the lower extremity of the sternum body. 1B: Sternal trepanation. 1C: Extrapleural intercostal incision using a curved tool passed through the sternal hole. 1D: Tube drainage passed through the extrapleural intercostal incision. 


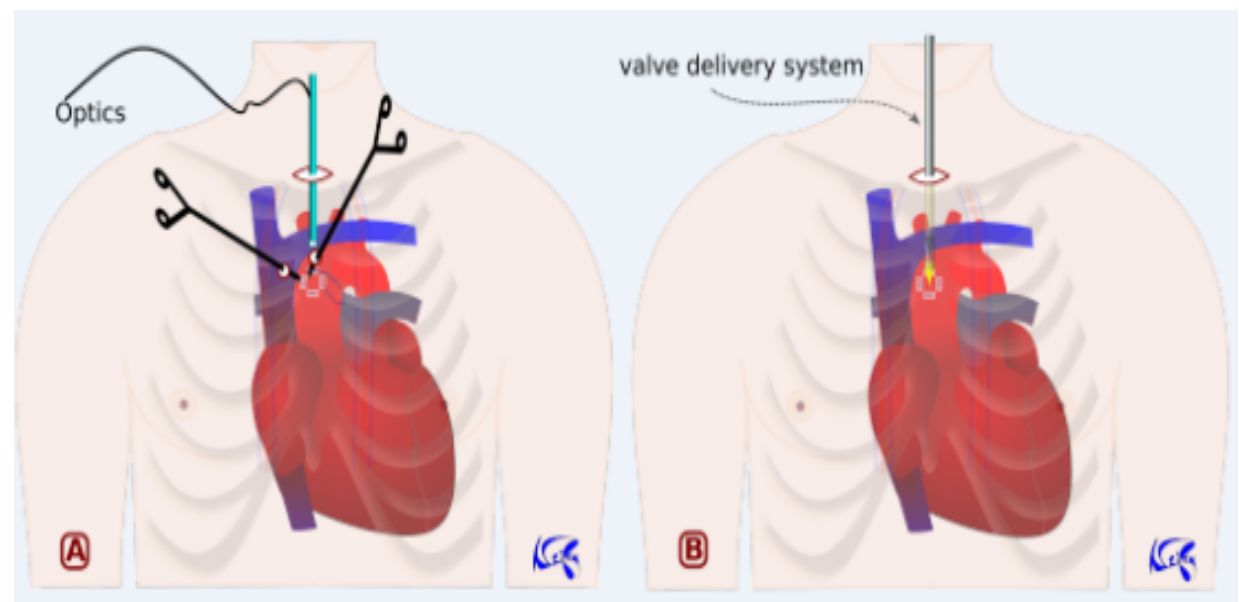

Figure 2. Neifer's approach for transaortic TAVI. 2A: Pledgeted aortic purse-strings sutures made proximal to the innominate artery using two working ports. 2B: Transcatheter valve delivery system introduced through the supra-sternal mini-incision.

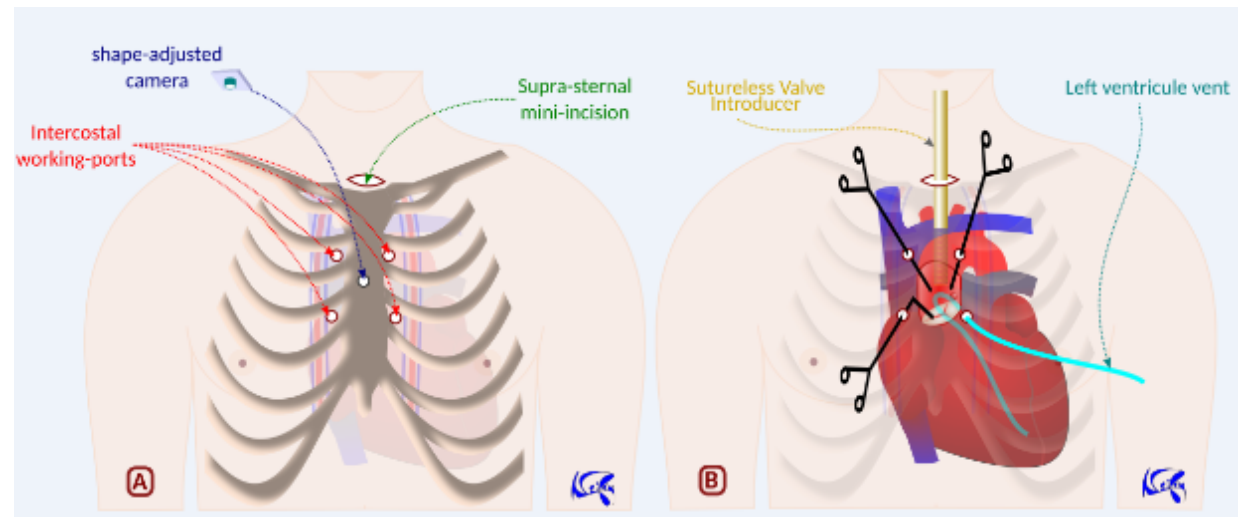

Figure 3. Neifer's approach for aortic valve surgery. 3A: Sternal hole (in the upper third of sternum body) with four concentric extrapleural intercostal ports. A suprasternal mini-incision may be useful to introduce aortic valve, optics and suction cannulas. Dedicated optics through the sternal hole may also be helpful. 3B: One port may be used for left ventricular venting. Through the other working ports, we can open aorta and resect aortic valve which will be replaced by a sutureless prosthesis introduced through the suprasternal incision.

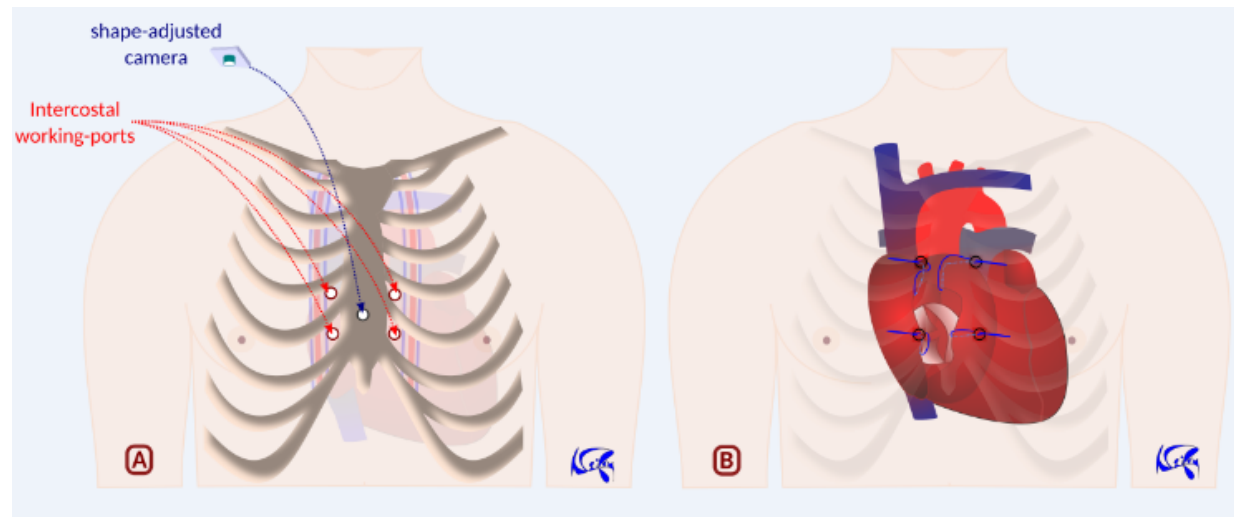

Figure 4. Neifer's approach for tricuspid valve surgery. 4A: Sternal hole (in the lower third of sternum body) with four concentric extrapleural intercostal ports. 4B: Right atrium is opened and spread by tracting wires passed through the intercostal incisions.

surgery through subxiphoid approach or thoracoscopy [5]. Using my approach, pericardial drainage will be even done, in critical situations, by the cardiologist or the emergency doctor indoors. An auto-stop electric cranial drill and a scalpel are all they need to save patient in less of one minute. We can imagine in the future these tools grouped as an "emergency kit for pericardial drainage" in all emergencies and intensive care units as well as defibrillators.
Percutaneous implantation of cardiac electronic devices (permanent pacemakers and implantable cardioverters defibrillators) is associated with a non-negligible rate of tricuspid valve dysfunction with subsequent increasing rate of mortality and heart failure hospitalisations [6]. Actual surgical approaches for epicardial leads implantation are generally used as second choice although development of video-assisted thoracoscopy. Using my procedure, we will be able to stimulate all cardiac chambers we need and will avoid 
disadvantages of both actual interventional and surgical techniques: no tricuspid valve damage, no $\mathrm{x}$-rays exposure, no limit to cardiac chambers stimulation, no chest pain due to thoracotomy, no chest drain due to pleura opening.

In a recent review of modalities of left ventricular unloading on extracorporeal life support [7], only $16 \%$ of cases are conducted surgically. Certainly, that can be explained by the aggressive character of actual surgical approaches which seem generally not suitable for patients in cardiogenic choc, pulmonary oedema, and anticoagulation therapy. Percutaneous approaches, however, are not usually effective and may be very expensive for some techniques (Impella ${ }^{\circ}$ for example). My approach proposes a comprehensive technique easy to do and cost effective.

First introduced in 2002 [8], Transcatheter Aortic Valve Implantation (TAVI) has since revolutionized treatment of aortic valve stenosis in risky patients. All procedures are actually undertaken under scopy and are mostly conducted by transfemoral approach. Transaortic access offers the advantage of a comfortable calibre to introduce the valve delivery system, but this approach, actually performed by ministernotomy or minithoracotomy, seems to be aggressive in elderly population. Radiation doses from TAVI are the highest used in interventional cardiac procedures, with potential harmful consequences [9]. Using my approach, there will be no radiation neither peripheral vascular complications in TAVI procedures.

Recently in 2016, a totally endoscopic suturless aortic valve implantation was successfully performed, using four right intrapleural intercostal incisions [10]. For this approach, a suitable anatomical presentation of aorta is needed. With my approach, access to the aortic valve will be allowed regardless of aorta conformation. Besides, access will be easier, offering better exposure and avoiding pleural opening and pulmonary exclusion.

Massive pulmonary embolism is a life-threatening condition with a high mortality rate exceeding $50 \%$ [11]. The best therapeutic management between thrombolysis, catheter directed fibrinolysis, and surgical embolectomy is still unclear [12]. Using my approach, pulmonary embolism will be easily treated avoiding the risk of major bleeding following thrombolysis and the morbidity of the conventional surgery.

In the last years, treatment of mitral valve disease has evolved using right three-port video-assisted thoracic surgery [13] and robotics [14]. For high-risk patients, multiples techniques for transcatheter repair or replacement have emerged [15]. Treatment of tricuspid valve is experiencing similar trend [16]. With my approach, surgical repair of both mitral and tricuspid valves will be endoscopically easy and feasible at the same procedure through the same incision of right atrium. Transcatheter technologies will be also allowed by direct puncture of right atrium without radiation exposure.

In a recent systematic review and meta-analysis, surgical treatment of atrial septal defects is associated with higher mortality and complications compared to percutaneous closure [17]. An endoscopic approach using a right mini-thoracotomy is recently reported to be effective with low morbidity and fast postoperative recovery [18]. This will be more evident with my approach which not necessitate thoracotomy.

As demonstrated, this innovative approach offers many advantages. However, its success needs to adapt working tools (surgical instruments with articulation ability under sternum, shape-adapted camera to the sternal hole allowing panoramic view, dedicated tools to implant adjusted bone substitute for sternal hole closure) and enhance procedures assessments from integrative preoperative exams to intraoperative augmented reality and live computer simulation. Ultimately, totally robotic cardiac surgery through this approach will become, I think, a close reality.

\section{Conclusion}

While this novel approach is still in progress, its contribution would be great allowing endoscopic and eventually robotic access to the cardiac cavity without chest, pleura, or sternum opening. This approach, allowing also transcatheter interventional procedures without X-rays use, would modify current practices and indications in both cardiac surgery and interventional cardiology.

\section{Acknowledgements}

First corpse experiments are in progress thanks to a great help of professor Bertrand Marcheix, chief of cardiac surgery department of Rangueil University Hospital, Toulouse, France.

\section{Conflict of interests}

I declare that I have no conflicts of interest related to this manuscript

\section{References}

1. Aris A (1996) One hundred years of cardiac surgery. Ann Thorac Surg 62: 636-637. [Crossref]

2. Ishikawa N, Watanabe G (2015) Robot-assisted cardiac surgery. Ann Thorac Cardiovasc Surg 21: 322-328. [Crossref]

3. Braimbridge MV (1993) The history of thoracoscopic surgery. Ann Thorac Surg 56 610-614. [Crossref]

4. Santana O, Xydas S, Williams RF, Wittels SH, Yucel E, et al. (2017) Minimally invasive valve surgery in high-risk patients. J Thorac Dis 9: S614-614S623. [Crossref]

5. Imazio M, Gaido L, Battaglia A, Gaita F (2017). Contemporary management of pericardial effusion: practical aspects for clinical practice. Postgrad Med 129: 178-186. [Crossref]

6. Chang JD, Manning WJ, Ebrille E, Zimetbaum PJ (2017) Tricuspid Valve Dysfunction Following Pacemaker or Cardioverter-Defibrillator Implantation. J Am Coll Cardiol 69: 2331-2341. [Crossref]

7. Meani P, Gelsomino S, Natour E, Johnson D, Rocca HB, et al. (2017) Modalities and Effects of Left Ventricle Unloading on Extracorporeal Life support: a Review of the Current Literature. Eur J Heart Fail 2: 84-91. [Crossref]

8. Cribier A (2012) Development of transcatheter aortic valve implantation (TAVI): A 20year odyssey. Archiv Cardiovas Dis 105: 146-152.

9. Gislason-Lee AJ, Keeble C, Malkin CJ, Egleston D, Bexon J, et al. (2016) Impact of latest generation cardiac interventional X-ray equipment on patient image quality and radiation dose for trans-catheter aortic valve implantations. Br J Radiol 89: 20160269. [Crossref]

10. Vola M, Fuzellier JF, Gerbay A, Campisi S (2016) First in Human Totally Endoscopic Perceval Valve Implantation. Ann Thorac Surg 102: e299-301. [Crossref]

11. Kucher N, Rossi E, De Rosa M, Goldhaber SZ (2006) Massive pulmonary embolism. Circulation 113: 577-582. [Crossref]

12. Edelman JJ, Okiwelu N, Anvardeen K, Joshi P, Murphy B, et al. (2016) Surgical Pulmonary Embolectomy: Experience in a Series of 37 Consecutive Cases. Heart Lung Circ 25: 1240-1244. [Crossref]

13. Ito T, Maekawa A, Hoshino S, Hayashi Y, Sawaki S, et al. (2017) Three-port (one incision plus two-port) endoscopic mitral valve surgery without robotic assistance. Eur $J$ Cardiothorac Surg 51: 913-918. [Crossref]

14. Cao C, Clark AL, Suri RM (2016) Robotic surgery is the optimal approach for mitra surgery. Ann Cardiothorac Surg 5: 563-566. [Crossref]

15. Sorajja P, Leon MB, Adams DH, Webb JG, Farivar RS (2017) Transcatheter Therapy for Mitral Regurgitation Clinical Challenges and Potential Solutions. Circulation 136: 404-417. [Crossref]

16. Brüstle K, Calen C, Kuwata S, Nietlispach F, Zuber M, et al. (2017) How to Treat Tricuspid Valve Disease: What's New on the Horizon? Curr Treat Options Cardiovasc Med 19: 18. [Crossref] 
Neifer C (2017) A novel approach in cardiac surgery: Neifer's approach

17. Villablanca PA, Briston DA, Rodés-Cabau J, Briceno DF, Rao G, et al. (2017) Treatment options for the closure of secundum atrial septal defects: A systematic review and metaanalysis. Int J Cardiol 241: 149-155. [Crossref]
18. Nishida H, Nakatsuka D, Kawano Y, Hiraiwa N, Takanashi S, et al. (2017) Outcomes of Totally Endoscopic Atrial Septal Defect Closure Using a Glutaraldehyde-Treated Autologous Pericardial Patch. Circ J 81: 689-693. [Crossref]

Copyright: (C2017 Neifer C. This is an open-access article distributed under the terms of the Creative Commons Attribution License, which permits unrestricted use, distribution, and reproduction in any medium, provided the original author and source are credited. 